\title{
Impact of early detection of acute invasive fungal rhinosinusitis in immunocompromised patients
}

\author{
Mariana L. C. Silveira', Wilma T. Anselmo-Lima', Francesca M. Faria², Danielle L. C. Queiroz', Rodrigo L. Nogueira', \\ Marcelo G. J. Leite ${ }^{1}$, Ricardo M. Lessa ${ }^{1}$, Belinda P. Simões ${ }^{3}$, Edwin Tamashiro ${ }^{1}$ and Fabiana C. P. Valera ${ }^{1 *}$ (D)
}

\begin{abstract}
Background: Early diagnosis of acute invasive fungal rhinosinusitis (AIFRS) is vital to improving outcomes in immunocompromised patients. This study evaluated the impact of a systematic protocol with nasal endoscopy and biopsies to early detect AIFRS in immunocompromised patients. Additionally, we compared the accuracy of frozensection biopsy and culture with formalin-fixed paraffin-embedded (FFPE) biopsy.

Methods: Retrospective cohort in a Tertiary Referral Hospital. Patients with the suspected diagnosis of AIFRS were evaluated following a standardized protocol, including serial nasal endoscopies and biopsies when necessary. The sensitivity and specificity of frozen-section biopsy and culture were also compared with FFPE.

Results: The mortality rate related to AIFRS of this standardized cohort (13/43) was 30.2\%. Better outcomes were observed in patients with disease limited to the turbinates and in those with higher peripheral neutrophils count. Frozen-section biopsy positivity correlated with FFPE findings for fungi detection ( $p$-value $<0.0001$ ), with a sensitivity of $90.6 \%$, specificity of $72.7 \%$, and accuracy of $86.0 \%$.

Conclusion: Implementation of this standardized protocol was related to a considerably low mortality rate among patients with suspected AIFRS at our Institution. Frozen-section biopsy revealed high accuracy to diagnose AIFRS. The current protocol including frozen-tissue biopsy improved the evaluation and survival rates of immunocompromised patients with presumed AIFRS.
\end{abstract}

Keywords: Rhinosinusitis, Acute invasive fungal rhinosinusitis, Fungi, Frozen-section biopsy, Paraffin-embedded paraffin, Accuracy, Mortality rate

\section{Background}

Acute invasive fungal rhinosinusitis (AIFRS) is a lifethreatening disease, affecting mostly immunocompromised patients with neutrophilic dysfunction [1-3]. In these patients, saprophytic fungi, particularly Zygomycetes and Aspergillus, can invade the nasal mucosa and blood vessels, leading to rapid dissemination into the orbits, palate and the brain [2-4]. For this reason, a systematic review states that the overall survival rate of AIFRS patients is as low as 50\% [2]. Early diagnosis and immediate

\footnotetext{
* Correspondence: facpvalera@fmrp.usp.br

'Department of Ophthalmology, Otorhinolaryngology, and Head and Neck Surgery, Ribeirão Preto Medical School, University of São Paulo, Av.

Bandeirantes, 3900 - $12^{\circ}$ andar., São Paulo, São Paulo CEP: 14049-900, Brazil

Full list of author information is available at the end of the article
}

treatment, including antifungal therapy and surgical debridement, are considered vital for better survival rates [2].

Unfortunately, patients with severe neutropenia usually present with vague symptoms (such as fever lasting for more than $48 \mathrm{~h}$ ) in early stages of AIFRS [5]. Other signs such as facial swelling or pain, proptosis, headache, seizures, or focal neurologic alterations are indicative of a later progression of this disease $[2,5]$, ultimately with poor prognosis $[2,6]$. Nasal endoscopy can reveal crusting, pale mucosa, or necrosis in the affected areas $[6,7]$.

Imaging workup is essential to evaluate extra-sinonasal extensiveness of the disease and for surgical planning. CT scans and MRI are necessary to evaluate bone and soft tissue involvements (i.e., orbit/brain), respectively 
[2, 8], although their findings in early stages are usually non-specific [7]. Unilateral disease and bone thickening on CT scans are highly suggestive of AIFRS [6]. Notably, some imaging tests are completely normal despite the endoscopic findings suggestive of AIFRS [9].

Formalin-fixed paraffin-embedded (FFPE) histopathological exam, including hematoxylin-eosin (HE) and Gomori methenamine-silver (GMS) staining, is the most reliable test to confirm fungi invasion into the tissue $[3,8,9]$. However, processing for FFPE is time-consuming, which contrasts with the urgency to diagnose AIFRS. To obtain faster results, some groups have advocated the inclusion of frozen-section biopsy [5, 9-12] in the evaluation of patients at risk. In most of these studies, frozen-section biopsy during surgery has demonstrated moderate to high sensitivity and specificity when compared with FFPE histopathology.

In a previous case-series from our service [6], we observed a mortality rate of $50 \%$ in 32 AIFRS patients. To improve our outcomes, we have implemented a new protocol for evaluation of neutropenic patients, which included serial nasal endoscopy (every $48 \mathrm{~h}$ ), associated with biopsy whenever appropriate.

The objective of this study was to investigate the impact of this protocol in the diagnosis and mortality rate of AIFRS, as well as to assess the sensitivity and specificity of frozen section biopsy in comparison with FFPE histopathology.

\section{Methods}

This is a retrospective study performed in a tertiary referral hospital (Clinics Hospital - Ribeirão Preto Medical School, University of São Paulo, Brazil). All patients with the suspected diagnosis of AIFRS were evaluated by the ENT team between January 2010 and December 2015.

In 2010, after a multidisciplinary meeting, (composed predominantly by ENTs and hematologists) we have implemented a more comprehensive protocol to screen AIFRS in patients under risk. Every immunocompromised patient with persistent fever of unknown origin (48 $\mathrm{h}$ or more, not responding to antibiotic therapy); sinonasal symptom - rhinorrhea, nasal obstruction, epistaxis, crusting; and/ or facial edema - was immediately referred from hematologists to the ENT team. All these patients underwent thorough physical examination, nasal endoscopy, complete blood count $(\mathrm{CBC})$ and sinonasal CT scan assessment (Fig. 1).

CT scans were scored according to a binary protocol (based on Lund-Mackay score), scoring "0" when the region was normal, and " 1 " if there was mucosal or bone thickening. The following sites were evaluated: maxillary sinus, anterior ethmoidal cells, posterior ethmoidal cells, frontal sinus, sphenoid sinus, and nasal cavity, with a possible maximum score of 12 points (6 points for each side).
Nasal endoscopy was performed in all patients, and we scored as "0" when normal or " 1 " when any suspicious mucosal alteration (pale area, darkened tissue, crust, ulcer or hyphae) was observed. Areas systematically evaluated during the nasal endoscopy were nasal septum, middle and inferior turbinates, lateral nasal wall, and nasopharynx. If any mucosa alteration was detected, three 5-mm specimens of the suspicious area were collected using nasal punch forceps: one for frozen-section biopsy, one for routine FFPE analysis (HE and GMS staining) and the third specimen for fungal culture. As routine in our service, complete blood count is performed daily in these patients. In severe thrombocytopenic patients (those with less than 20,000 platelets $/ \mathrm{ml}$ ), nasal endoscopy and biopsy were performed immediately after platelet transfusion. In all other patients, biopsies were obtained without major complications. Some patients received cotton embedded with vasoconstrictor to contain local bleeding.

Two samples were immediately sent to the Pathology Laboratory. The first was submitted to frozen-section biopsy, following a standardized protocol. Briefly, the specimen was immersed in Tissue-Tek medium at Freezing Microtome (Microm-HM-525), at $-28^{\circ} \mathrm{C}$. Histological sections of 4-5 $\mu \mathrm{m}$ were obtained, colored with Hematoxylin-Eosin, and evaluated with an Olympus-BX51 microscope, to assess the presence of fungi. The mean time for this analysis was about $30 \mathrm{~min}$ (Fig. 2a).

The other sample was submitted to FFPE testing. The sample was fixed in $10 \%$ buffered-formalin and processed by an automatic tissue processor. The specimens embedded in paraffin were sectioned $(4-5 \mu \mathrm{m})$, stained with HE and GMS, and analyzed for fungal presence and invasiveness of the mucosa. The time for this analysis in our service was about 5-7 days (Fig. 2b).

The third sample was sent to the mycology laboratory and cultured on Sabouraud medium. The mean time for a positive result was 7 days.

Patients with positive nasal biopsies (confirmed AIFRS) or those with highly suggestive symptoms/signs of AIFRS underwent surgical debridement and systemic antifungal therapy (Fig. 1). Surgical debridement was mainly performed by endoscopic sinus surgery (ESS), associated with open surgeries whenever orbital, intracranial, or palatal involvement was observed. During this procedure, new samples were obtained for frozen-section, FFPE biopsies, and micology analysis. Immediately after diagnosis, systemic liposomal amphotericin-B or voriconazole was started. Patients were then periodically evaluated at the ENT clinic with nasal endoscopy (every 24-48 h) until neutropenia was recovered and no sinonasal symptoms and signs were present (Fig. 1).

Data regarding nasal endoscopic findings, associated comorbidities, $\mathrm{CBC}$, CT scan evaluation, biopsy, and 


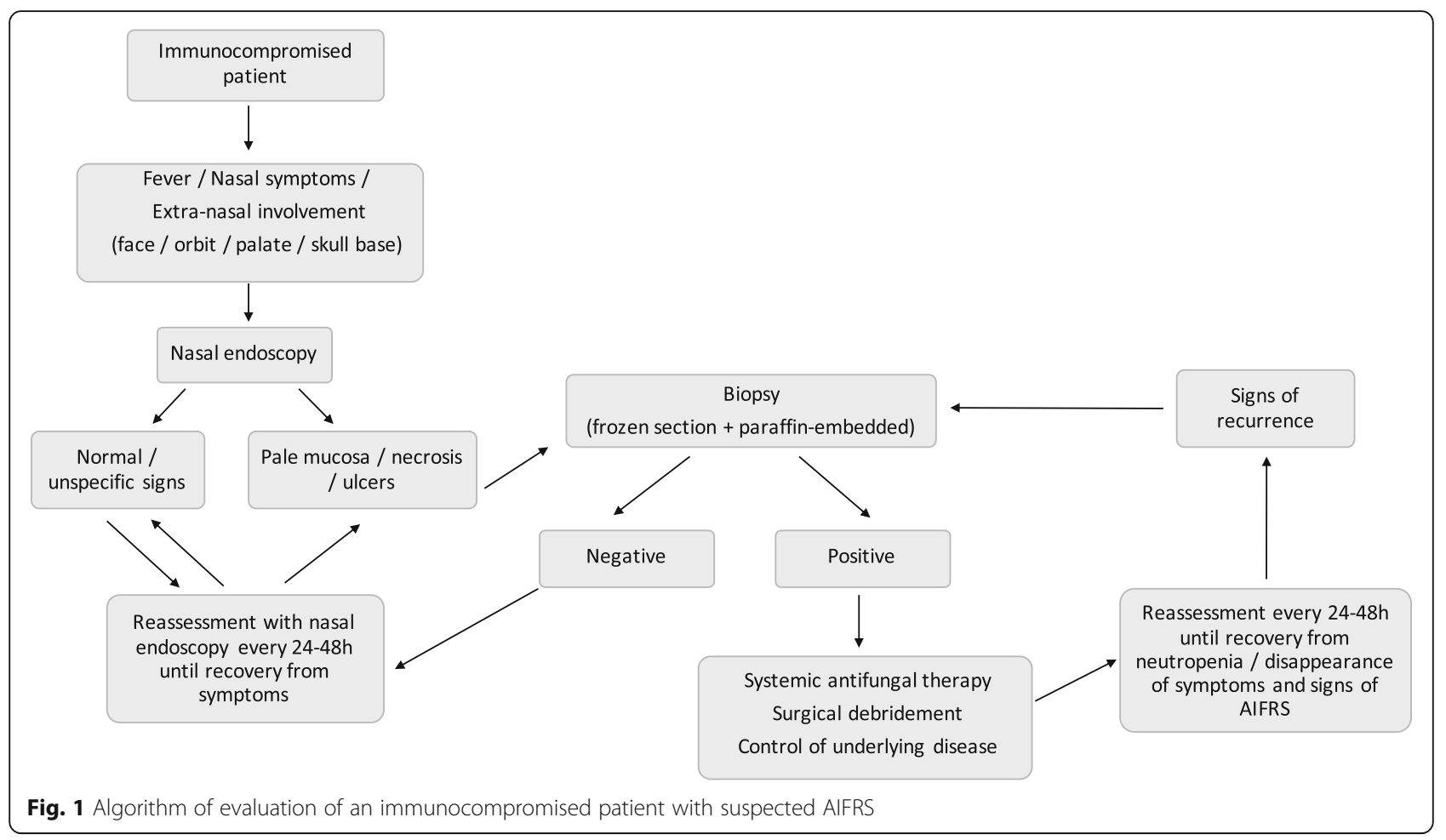

culture were obtained from medical records. Patient outcomes were assessed to calculate mortality rate related to the fungal disease. Patients with incomplete clinical data were excluded from the study.

This study was approved by the local IRB (Clinics Hospital - Ribeirão Preto Medical School - University of São Paulo), under the number of CAAE 62294116.6.000 0.5440 . As this was a retrospective cohort, and data was collected from medical records, exemption to obtain informed consent was demanded to local IRB, and it was considered approved.

\section{Statistical analysis}

The mortality rate from the cohort was described, considering the disease-related mortality, i.e. the unfavorable outcome when patients died explicitly from invasive fungus infection either sinonasal or pulmonary.

The clinical factors of both groups (surviving patients vs. dead ones) were compared using the unpaired Student t-test or with Fisher's exact test. Fisher's exact test was also used to evaluate the association between FFPE biopsy with frozen section biopsy and culture. Sensitivity, specificity, positive predictive value (PPV), and negative predictive values (NPV) were assessed, considering both frozen-section biopsy and culture as index tests, and paraffin-embedded biopsy as the reference standard test [13]. For all the statistical analyses, difference was considered significant when $p$-value $<0.05$.

\section{Results}

Forty-six patients with the suspected diagnosis of AIFRS were evaluated during the period. Three patients were excluded because they did not have complete data in their files.

Among the remaining 43 patients, 23 were male; and age ranged from 1 to 70 years (mean: $40.1 \pm 20.4$ ). The following associated diseases were present: hematological malignancies in 25 patients (58.2\%); aplastic anemia in 11 (25.6\%), autoimmune disorders in $3(7 \%)$, diabetes mellitus in $2(4.6 \%)$, HIV infection in $1(2.3 \%)$ and chronic renal failure in $1(2.3 \%)$.

Thirteen patients (30.2\%) died of invasive fungal infection: in 11 cases, AIFRS was the cause of death; another two died due to pulmonary Aspergillosis. The associated diseases among the patients that died were hematological malignancies $(n=8)$, aplastic anemia $(n=3)$, diabetes $(n=1)$, and autoimmune disorder $(n=1)$. The distribution of mortality regarding the primary disease was not significant $(p$-value $=1.00)$.

Among the patients with hematological diseases (aplastic anemia or hematological malignancies, $n=36$ ), the average time of neutropenia when AIFRS was diagnosed was 35 days. These patients exhibited white blood cell count of $850 \pm 800$ cells $/ \mu \mathrm{L}$ and absolute neutrophil count of $330 \pm 200$ cells $/ \mu \mathrm{L}$. The remaining seven patients had a different primary disease, without history of neutropenia or decreased white blood cell count at the evaluation. 




Fig. 2 Histological sections of a patient with AIFRS, showing extensive necrosis, and abundance of hyphae invading mucosa with vascular embolization. Paucity septation with predominant acute angle division suggests Aspergillus species. 2a) Frozen-section section hematoxylineosin stain, 100x; 2b) Paraffin-embedded slide, hematoxylin-eosin stain, 100x; 2c) Paraffin-embedded slide, Gomori-methanamine-silver stain, 100x

Neither the length of neutropenia ( $45.7 \pm 18$ vs. $33.8 \pm$ 8 days, $p$-value $=0.55)$ nor the absolute white blood cell count $(1.1 \pm 0.2$ vs. $0.7 \pm 0.3$ cells $/ \mu \mathrm{L}, p$-value $=0.48)$ was different between the groups who lived and the one that died, respectively. Nonetheless, the level of neutropenia was significantly lower in patients who died $(0.2 \pm 0.1$ cells $/ \mu \mathrm{L})$ when compared with the patients alive $(1.8 \pm$ 0.7 cells $/ \mu \mathrm{L}, p$-value $=0.04$ ).

During the first nasal endoscopy, 31 patients presented pale or darkened mucosa only in the middle or inferior turbinates. Among them, eight patients died (mortality rate $=25 \%$ ). In contrast, 7 of 12 patients who presented with fungal disease in the lateral wall or nasal septum died (mortality rate $=58 \% ; p=0.07$ ).

On CT scan, the most affected areas were the middle meatus, ethmoid, and the maxillary sinus (respectively in 31, 25 and 27 patients). Frontal and sphenoid sinuses were affected in 14 patients. Five patients presented extra-nasal extension on CT scans, four in orbit and one intracranially. Disease extension on CT scan was not related to the mortality rate $(p=0.47)$. Notably, five patients (11.6\%) presented a total CT scan score of "0", meaning that they did not have any sinonasal change on CT scans. Additionally, other 12 patients (27.9\%) presented a total score of " 1 " or "2", indicating minimal changes on CT scans.

Thirty-five patients presented positive biopsies at ENT evaluation, undergoing surgery as soon as medical conditions were allowed. In 8 patients, both frozen and paraffin-embedded biopsies were negative, and they were followed-up with nasal endoscopy every 24-48 h. In 6 of them, symptoms and signs were controlled clinically, with no confirmation of AIFRS; other two patients persisted with fever and changes in nasal endoscopy, and surgery was indicated regardless the initial biopsies. Surgery performed was the endoscopic debridement of all involved areas in sinonasal areas (37 patients), associated with orbital exenteration in 4 patients and intracranial debridement in 1 patient.

After surgery, all patients were submitted to scheduled evaluation with nasal endoscopy. As soon as a pale/necrotic area were observed, they were reassessed with a new ESS debridement. Fourteen patients (32.5\%) underwent multiple procedures. The number of surgeries for those patients who died was similar with those who were alive (respectively $1.7 \pm 0.3$ vs. $1.3 \pm 0.2$, p-value $=0.20$ ).

The positivity-rate in both frozen-section and FFPE biopsies are shown in Table 1 . The sensitivity of frozen-section biopsy was $90.6 \%$; specificity was $72.7 \%$;

Table 1 Distribution of patients $(n=43)$ regarding the positivity rate in frozen-section and formalin-fixed paraffin-embedded (FFPE) biopsies

\begin{tabular}{lll}
\hline & $\begin{array}{l}\text { Positive FFPE } \\
\text { biopsy }\end{array}$ & $\begin{array}{l}\text { Negative FFPE } \\
\text { biopsy }\end{array}$ \\
\hline Positive Frozen-section biopsy & 29 & 3 \\
Negative Frozen-section biopsy & 3 & 8 \\
\hline
\end{tabular}


PPV was 90.6\%; NPV was $72.7 \%$; and accuracy was $86.0 \%$ ( $p$-value $<0.0001)$.

Data comparing culture with FFPE biopsy were available in 29 patients. Fungal culture was positive in 6 cases for Aspergillus ssp., 3 for Mucor, and 1 for Histoplasma $s p$. The agreement between culture and FFPE biopsy was not significant $(p=0.62)$. Sensitivity of culture was $80.0 \%$; specificity was $40.0 \%$; PPV was $21.0 \%$; NPV was 90.9\%; and accuracy was $55.2 \%$ (Table 2).

The mortality rate of this current standardized cohort was $30.2 \%(n=13 / 43)$.

\section{Discussion}

The early diagnosis of AIFRS is challenging and demands accurate and fast tests [14]. With this aim, we have established a protocol to maximize the sensitivity to diagnose AIFRS, in such a way that patients under risk, with subtle or even with non-specific symptoms, are immediately referred by hematologists to ENT evaluation. During this exam, to increase sensitivity in the diagnosis, we have elected nasal endoscopy as the essential exam, followed by biopsy, instead of CT findings.

Actually, as we observed in our current cohort, a significant percentage of patients with confirmed AIFRS (39.5\%) had CT scans with few or even normal findings. Our data reinforce that nasal endoscopy must be elected as the preferred choice to evaluate patients at risk because it can reveal subtle changes in the nasal mucosa $[6,14,15]$ that are not detected by imaging exams $[6,15]$. Taking into consideration only CT scans findings, these patients would have experienced a delayed treatment, and this could negatively impact on their outcome.

After the implementation of this protocol, we have been able to diagnose AIFRS in a faster and more accurate way. As a consequence, patients are undergoing surgery in early stages of the disease, with restricted sinonasal involvement. For instance, the majority of cases $(72.1 \%)$ had invasiveness restricted to the turbinates, mainly the middle turbinate. The combination of limited invasiveness of the disease at the diagnosis with a careful post-operative follow-up was related to a good outcome (30.2\% of disease-related deaths) when compared to the literature $[2,6,12,14,15,18]$.

Another contribution to detect AIFRS in early stages was the performance of frozen-section biopsies $[3,4,10,15,16]$, the presence of artifacts during the

Table 2 Distribution of patients ( $n=29)$ regarding the positivity rate in culture and formalin-fixed paraffin-embedded (FFPE) biopsies Positive FFPE biopsy Negative FFPE biopsy

\begin{tabular}{lll}
\hline Positive Culture & 10 & 1 \\
Negative Culture & 12 & 6 \\
\hline
\end{tabular}

freezing processing is still concerning. In our cohort, we observed that frozen-section and paraffin-embedded biopsies were well correlated (accuracy of $86 \%$ ). Our accuracy is quite similar to previous reports in literature [5, 9-11], showing high sensitivity and moderate specificity. However, three patients in our cohort presented positive frozen-section but negative paraffin-embedded biopsy, and this might have occurred because in our study small samples were taken under local anesthesia in awake patients for the frozen-section and paraffin-embedded biopsies, while in other studies they were obtained intra-operatively $[5,9,10]$. To the best of our knowledge, the present study is the largest series of patients that specifically used frozen-section biopsy for the diagnosis of AIFRS.

Mycological tissue culture has also been considered helpful in evaluating AIFRS because it can determine the fungi species and direct the antifungal therapy. However, it does not show invasiveness of mucosa and, therefore, cannot establish the definitive diagnosis of AIFRS. Moreover, it usually takes a minimum of 7 days to obtain the results, and a significant number of negative results is expected [10, 17]. For instance, only 11 patients (36\%) in our recent cohort had positive culture results. The association between culture results and paraffin-embedded biopsy was not significant, with low accuracy of $55.2 \%$.

The cause of immunodeficiency was not related to prognosis in our cohort and this may be due to the limited number of patients without hematological diseases. In this specific population, the low number of neutrophils was negatively related to the survival rate. This agrees with several other studies [2,14, 18], pointing out that neutropenia might be one of the most critical risk factors for the development and prognosis of AIFRS.

The surgical procedure associated with prompt initiation of antifungal therapy is critical for the outcome of AIFRS. In our protocol, patients were carefully followed-up with periodic nasal endoscopy after surgery, and every new alteration was determinant to indicate a further surgical debridement. In our series, we observed that $32.5 \%$ of patients needed at least one revisional surgery, similarly as reported by Payne et al. [14]. As important as the surgery itself, we believe that the continuous follow-up might have contributed to the better outcomes in these patients [2].

In summary, we observed that frozen-section biopsy is a fast and reliable exam to confirm the diagnosis of fungal invasion, with good accuracy when compared to the gold-standard FFPE biopsy.

Most importantly, the present protocol, based on direct suspicion by hematologists, comprehensive screening by ENTs and follow-up with nasal endoscopy in patients under risk, was associated with a considerable decrease in our Institutional mortality rate related to AIFRS, from 
50 to $30.2 \%$. This algorithm reinforces that nasal endoscopy should be preferred to CT scans to identify AIFRS since almost $40 \%$ of the patients in this cohort presented normal CT images. Finally, the constant pursuit of a precise AIFRS identification, either at primary diagnosis or at recurrence, is essential for improving outcome in immunocompromised patients.

\section{Conclusions}

The present protocol, based on an immediate evaluation by ENTs in patients under risk of AIFRS, was associated with a considerable decrease in mortality rate related to AIFRS in our hospital. Also, we observed that frozensection biopsy is a fast and reliable exam to confirm the diagnosis of fungal invasion, with good accuracy when compared to the gold-standard FFPE biopsy.

\section{Abbreviations}

AIFRS: Acute invasive fungal rhinosinusitis; ENT: Ear nose and throat specialist; FFPE: Formalin-fixed paraffin-embedded

\section{Acknowledgments}

The authors thank Mariane S. Yui and Andréa A. B. Biagiotti for their help with the revision of the medical records.

\section{Funding}

As a retrospective analysis, this study did not need financial support.

\section{Availability of data and materials}

The dataset used and/or analysed during the current study are available from the corresponding author on reasonable request.

\section{Authors' contributions}

MLCS and DLCQ collected the data; MLCS also participated on statistical analysis and on manuscript writing; FMF participated on histological analysis; RLN, MGJL, and RML helped with the execution of the new protocol; WTAL and BPS helped with the development of this new protocol, and with its execution; ET and FCPV helped with the development of this new protocol, with its execution, and with manuscript writing; FCPV also helped with statistical analysis and with data arrangement. All the authors have read and approved this final version of the manuscript.

\section{Ethics approval and consent to participate}

This study was approved by the local IRB, under the number of CAAE 62294116.6.0000.5440. As this was a retrospective cohort, and data was collected from medical records, exemption to obtain informed consent was demanded to local IRB, and it was considered approved.

\section{Consent for publication}

$$
\text { Not applicable }
$$

\section{Competing interests}

The authors declare that they have no competing interests.

\section{Publisher's Note}

Springer Nature remains neutral with regard to jurisdictional claims in published maps and institutional affiliations.

\section{Author details}

'Department of Ophthalmology, Otorhinolaryngology, and Head and Neck Surgery, Ribeirão Preto Medical School, University of São Paulo, Av. Bandeirantes, 3900 - $12^{\circ}$ andar., São Paulo, São Paulo CEP: 14049-900, Brazil. 2Division of Pathology, Clinics Hospital of Ribeirão Preto Medical School, University of São Paulo, São Paulo, Brazil. ${ }^{3}$ Department of Internal Medicine, Division of Hematology, Ribeirão Preto Medical School, University of São Paulo, São Paulo, Brazil.
Received: 23 March 2018 Accepted: 25 March 2019

Published online: 05 April 2019

\section{References}

1. Deshazo RD. Syndromes of invasive fungal sinusitis. Med Mycol. 2009; 47(Suppl 1):S309-14. https://doi.org/10.1080/13693780802213399.

2. Turner JH, Soudry E, Nayak JV, Hwang PH. Survival outcomes in acute invasive fungal sinusitis: a systematic review and quantitative synthesis of published evidence. Laryngoscope. 2013;123:1112-8. https://doi.org/10. 1002/lary.23912

3. Chakrabarti A, Denning DW, Ferguson BJ, Ponikau J, Buzina W, Kita H, et al. Fungal rhinosinusitis: a categorization and definitional schema addressing current controversies. Laryngoscope. 2009;119:1809-18. https://doi.org/10. 1002/lary.20520

4. Ruhnke M, Böhme A, Buchheidt D, Cornely O, Donhuijsen K, Einsele $H$, et al. Diagnosis of invasive fungal infections in hematology and oncologyguidelines from the infectious diseases working party in Haematology and oncology of the German Society for Haematology and oncology (AGIHO). Ann Oncol. 2012;23:823-33. https://doi.org/10.1093/annonc/mdr407.

5. Melancon CC, Clinger JD. The use of frozen section in the early diagnosis of acute invasive fungal sinusitis. Otolaryngol Neck Surg. 2017;157:314-9. https://doi.org/10.1177/0194599817697279.

6. Valera FCP, do LT, Tamashiro E, Yassuda CC, Silveira F. Anselmo-Lima WT Prognosis of acute invasive fungal rhinosinusitis related to underlying disease. Int J Infect Dis. 2011;15:841-4. https://doi.org/10.1016/j.jijid.2011.08.005.

7. Thompson GR, Patterson TF. Fungal disease of the nose and paranasal sinuses. J Allergy Clin Immunol. 2012;129:321-6. https://doi.org/10.1016/j. jaci.2011.11.039.

8. Dwyhalo KM, Donald C, Mendez A, Hoxworth J. Managing acute invasive fungal sinusitis. J Am Acad Physician Assist. 2016;29:48-53. https://doi.org/ 10.1097/01.JAA.0000473374.55372.8f.

9. Papagiannopoulos P, Lin DM, Al-Khudari S, Rajan K, Reddy S, Gattuso P, et al. Utility of intraoperative frozen sections in surgical decision making for acute invasive fungal rhinosinusitis. 2017;0:1-6. https://doi.org/10.1002/alr.21918.

10. Ghadiali MT, Deckard NA, Faroog U, Astor F, Robinson P, Casiano RR. Frozen-section biopsy analysis for acute invasive fungal rhinosinusitis. Otolaryngol - Head Neck Surg. 2007;136:714-9. https://doi.org/10.1016/j. otohns.2007.01.002.

11. Taxy JB, El-Zayaty S, Langerman A. Acute fungal sinusitis: natural history and the role of frozen section. Am J Clin Pathol. 2009;132:86-93. https://doi.org/ 10.1309/AJCP9HTH9NRPMYCT.

12. Foshee J, Luminais C, Casey J, Farag A, Prestipino A, lloreta AM, et al. An evaluation of invasive fungal sinusitis outcomes with subsite analysis and use of frozen section analysis. Int Forum Allergy Rhinol. 2016;6:807-11. https://doi.org/10.1002/alr.21714.

13. Bossuyt PM, Reitsma JB, Bruns DE, Gatsonis CA, Glasziou PP, Irwig L, et al. STARD 2015: an updated list of essential items for reporting diagnostic accuracy studies. Clin Chem. 2015;61:1446-52. https://doi.org/10.1373/ clinchem.2015.246280

14. Payne SJ, Mitzner R, Kunchala S, Roland L, McGinn JD. Acute invasive fungal rhinosinusitis: a 15-year experience with 41 patients. Otolaryngol - Head Neck Surg. 2016;154:759-64. https://doi.org/10.1177/0194599815627786.

15. Gillespie MB, O'Malley BW. An algorithmic approach to the diagnosis and management of invasive fungal rhinosinusitis in the immunocompromised patient. Otolaryngol Clin N Am. 2000;33:323-34

16. deShazo RD, O'Brien M, Chapin K, Soto-Aguilar M, Gardner L, Swain R. A new classification and diagnostic criteria for invasive fungal sinusitis. Arch Otolaryngol Neck Surg. 1997;123:1181-8. https://doi.org/10.1001/archotol. 1997.01900110031005

17. Badiee P, Moghadami M, Rozbehani H. Comparing immunological and molecular tests with conventional methods in diagnosis of acute invasive fungal rhinosinusitis. J Infect Dev Ctries. 2016;10:90-5. https://doi.org/10. 3855/jidc.6411.

18. Cho H-J, Jang M-S, Hong SD, Chung S-K, Kim HY, Dhong H-J. Prognostic factors for survival in patients with acute invasive fungal rhinosinusitis. Am J Rhinol Allergy. 2015;29:48-53. https://doi.org/10.2500/ajra.2015.29.4115. 